\title{
Moulin Rouge (2001) en la formación cultural de estudiantes de medicina
}

\section{Daniel Sebastián GARCÍA TORRES}

Facultad de Medicina No. 1 Universidad de Ciencias Médicas de Santiago de Cuba (Cuba).

Autor para correspondencia: Daniel García Torres. Correo electrónico: danielgarcia@infomed.sld.cu

Recibido el 27 de abril de 2018; aceptado el 16 de mayo de 2018.

Cómo citar este artículo: García Torres DS. Moulin Rouge (2001) en la formación cultural de estudiantes de medicina. Rev Med Cine [Internet] 2019;15(1): 33-36. DOI: $\underline{\text { http://dx.doi.org/10.14201/rmc.20243 }}$

\section{Resumen}

La formación de médicos en el presente siglo XXI, ameritan de una educación cultural que estimule el conocimiento artístico y científico, en la cual el cine se vincula como un ente facilitador al docente y al individuo para lograr un influjo de su práctica profesional que eleve la pertinencia de su gestión y de la institución pública encargada de la salud, en este sentido sobresalen filmes como Moulin Rouge (2001) que bien empleado procrea, mediante el debate, el aprendizaje creativo de los estudiantes quienes desempeñan un papel más activo en la búsqueda del conocimiento a partir de las situaciones complejas de la vida que se reflejan en el material cinematográfico.

Palabras clave: Moulin Rouge (2001), filmes, cultura, salud, estudiantes de medicina.

\section{Moulin Rouge (2001) in the cultural formation of students of medicine}

\section{Summary}

The formation of doctors presently XXI century, they drive to a cultural education that stimulates the artistic and scientific knowledge, in which the cinema is linked as an entity facilitator to the educational one and the individual to achieve a projection of its professional practice that elevates the relevancy of its administration and of the public institution in charge of the health, in this sense they stand out films like Moulin Rouge (2001) that very employee stimulates, by means of the debate, the creative learning of the students who you/they play a more active part in the search of the knowledge starting from the complex situations of the life that are reflected in the film material.

Keywords: Moulin Rouge (2001), Film, Culture, Health, Medicine students.

El autor declara que el artículo es original y que no ha sido publicado previamente. 
Introducción

"En la vida los más importante es siempre sentir amor y ser amado a cambio", así comienza una singular historia de amor erigida en todo un espectáculo musical y a la vez un material de incalculable valor didáctico: Moulin Rouge (2001) de Baz Luhrmann.

Otras producciones como Chicago (2002) conducida por Rob Marshall, basada en un musical de teatro estrenado con el mismo título en los años 70; Sweeney Todd, el barbero demoníaco de la calle Fleet / Sweeney Todd: The Demon Barber of Fleet Street (2007) de Tim Burton, adaptación cinematográfica del musical homónimo de Stephen Sondheim y Hugh Wheeler o Los miserables / Les Misérables (2012) de Tom Hooper que recrea la obra magistral de Victor Hugo publicada en 1862, son connotados ejemplos de buen arte cuya promoción y debate entre los jóvenes médicos conduce a elevar su cultura.

Es importante que las proyecciones cinematográficas sean sometidas a un análisis crítico en el que estos jóvenes tengan la oportunidad de exponer sus criterios y el carácter reflexivo de sus pensamientos con una óptica novedosa y que guie a proyecciones futuras en la praxis médica.

En esta propuesta se aborda la utilización de la película Moulin Rouge en polémica con estudiantes de Medicina y su impacto en la cultura general adecuado a la pertinencia social del currículo de la carrera.

Ficha técnica

Título original: Moulin Rouge.

País: Australia.

Año: 2001.

Director: Baz Luhrmann.

Música: Craig Armstrong.

Fotografía: Donald McAlpine.

Montaje: Jill Bilcock.

Guion: Craig Pearce, Baz Luhrmann.

Intérpretes: Nicole Kidman, Ewan McGregor, John Leguizamo, Jim Broadbent, Richard Roxburgh, David Wenham, Jacek Koman, Kylie Minogue, ...

Color: color.

Duración: 125 minutos.

Género: musical, drama romántico.

Idioma original: inglés.

Productora: 20th Century Fox, Bazmark Films.

Sinopsis: ambientada en el París bohemio de
1900. Satine, la estrella más rutilante del Moulin Rouge, encandila a toda la ciudad con sus bailes llenos de sensualidad y su enorme belleza. Atrapada entre el amor de dos hombres, un joven escritor y un duque, lucha por hacer realidad su sueño de convertirse en actriz. Pero, en un mundo en el que todo vale, excepto enamorarse, nada es fácil.

Premios: Oscar a la Mejor dirección artística y Vestuario. Nominada al Oscar a la Mejor Película, Actriz Principal, Fotografía, Montaje, Maquillaje y Sonido (2002).

Enlaces:

https://www.filmaffinity.com/es/film255392.html

https://www.imdb.com/title/tt0203009

\section{$\underline{\text { Trailer en español }}$}

Potencialidades del filme Moulin Rouge en la educación de nuevas generaciones de médicos

En la enseñanza de Medicina se ha incorporado el análisis sobre las potencialidades que brinda el séptimo arte para educar e incluso sanar brindando un acercamiento preventivo hacia la sociedad $1,2,3,4$.

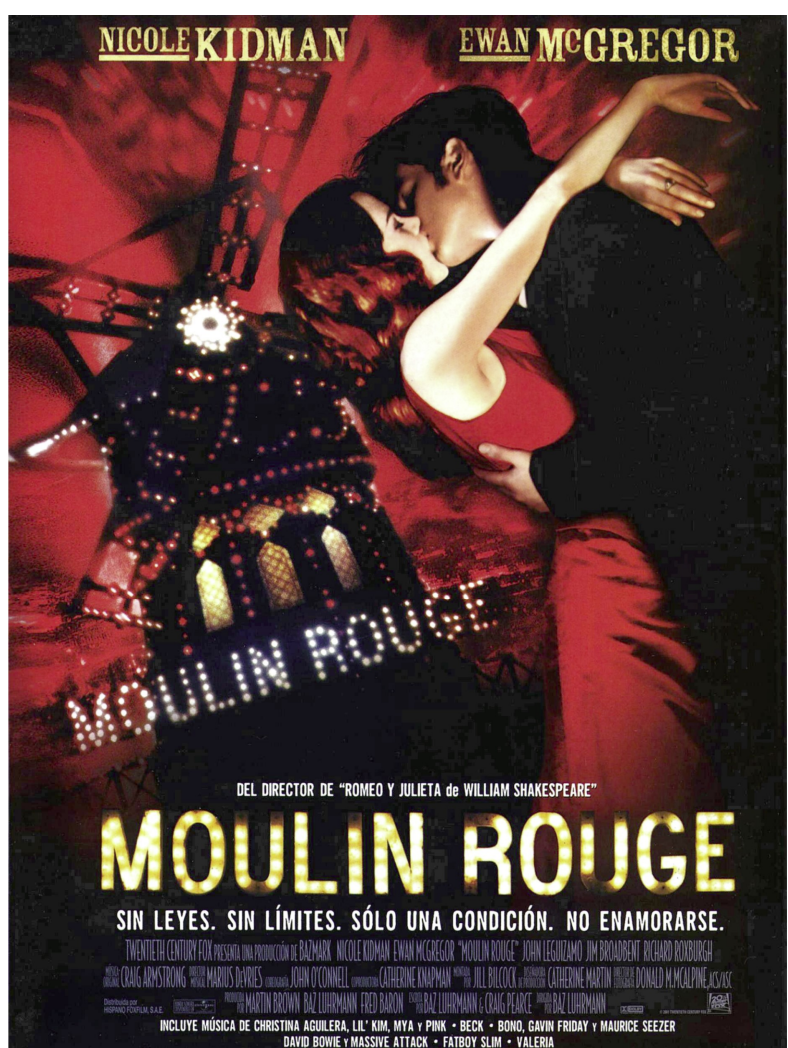


El debate de propuestas de la cinematografía mundial dirigido a estudiantes de Medicina, constituye una forma idónea de utilizar los filmes en función de una educación artística y provisora, capaz de incidir en la consolidación de valores éticos y morales. Sobre todo, cuando se hace necesario dotar estos con una educación artística apreciativa que provea una respuesta profesional más rica, sostenida por la tradición y la universalidad de lo cultural ${ }^{5}$.

Algunos autores proponen incluir la salud como un valor moral en la concepción curricular pues se debe entender como un valor del ser humano, y reclama la construcción de una cultura en torno a ella6.

En esta dirección se propone reflexionar también sobre el concepto de cultura sobre todo si es concebido como el conjunto de conocimientos adquiridos por los pueblos, empleado para interpretar experiencias sobre objetos, acciones o eventos y generar conductas sociales 7 .

Precisamente el cine percibe elementos que posibilitan asimilar contenidos de la cultura general con un enfoque dirigido a la salud beneficiado por una estrecha relación con su aplicación bioética y la destreza médica, favoreciendo que los estudiantes puedan tener un acercamiento efectivo al aprendizaje mediante la visualización e intercambios sobre esta, en los cuales debe predominar la exploración, el discernimiento y la investigación.

El resultado didáctico de las proyecciones audiovisuales proporciona una adecuada correlación entre regularidades y teorías de la ciencia con la vida y de la actividad práctica con el pensamiento abstracto, procesos culturales bien apreciados en la formación humanística del doctor contemporáneo.

El lenguaje del cine acondiciona al individuo para percibir el máximo de sensaciones, percepciones y representaciones que se aportarán al proceso de educación y especialización llegando incluso a aportar un conjunto de emociones fuertes y variadas que conlleva a disfrutar de la búsqueda científica.

La visualización de una película pone en marcha los sistemas perceptivos (vista y oído), a los que se suman otros lenguajes asociados, como la música y los efectos especiales que hacen del lenguaje cinematográfico uno de los más ricos y complejos en los que el docente puede incidir con contenidos sobre salud, en dependencia de las metas trazadas y la planificación, organización y evaluación del proceso. El cine es una herramienta valiosa para el estudio de situaciones trascendentales, tales como el dolor, la enfermedad y la muerte ${ }^{8}$.

En esta experiencia se asume el concepto cultura socio - médica como proceso de reconstrucción constante de saberes y procederes que sustentados en significados a los que el profesional médico le confiere sentido y le posibilita a este desarrollar, preservar y difundir la salud en el hombre dado que en su accionar y proyección formativa valores, creencias, ideología, costumbres, ideales, producto de las diversas historias sociales y culturales de cada uno de los ambientes en que recrea su destreza competitiva9.

En Moulin Rouge, se recrean aspectos que posibilitan actuar con un carácter preventivo e instructivos con los estudiantes y con la población como la pérdida de valores en una sociedad degradada, prostituida, en la que se abusa del alcohol y los jóvenes son pervertidos por un mundo desigual en el cual al talento le cuesta mucho trabajo imponerse como le es difícil al amor - en este caso con el escritor Christian encarnado por Ewan McGregor - prevalecer por encima de ellos sobre todo frente al poder corruptor de su antagonista el Duque de Monroth desentrañado por Richard Roxburgh símbolo de la decadencia pero muy presente en la vida cotidiana aunque sin título de nobleza pero con cierta jerarquía, cargo político o posición económica (Foto 1 y 2).

Satine, la protagonista interpretada por la bella Nicole Kidman, - de quien resalta su exquisita femineidad que vuelve a mostrarnos 16 años después en $E I$ sacrificio de un ciervo sagrado / The Killing of a Sacred Deer (2017) de Giorgos Lanthimos - es un ejemplo de esta existencia competitiva, ella expresa: "solo valgo lo que me paguen". Triste realidad vigente en la modernidad.

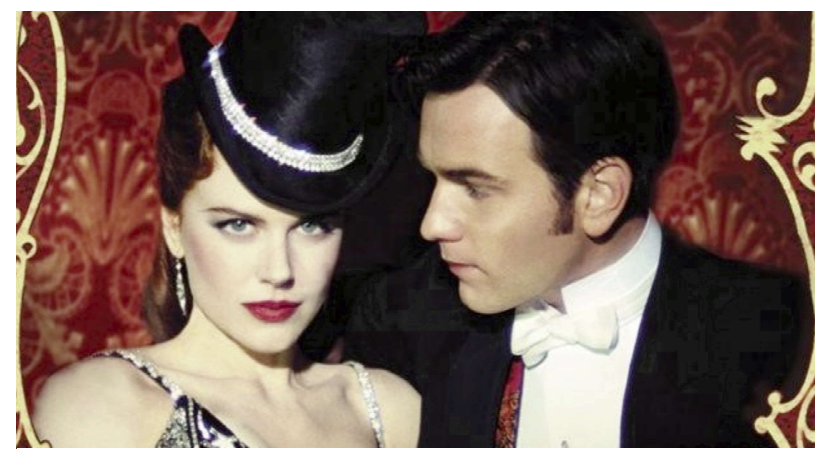

Foto 1. Satine (Nicole Kidman) en danza de amor con Christian (Ewan McGregor. 


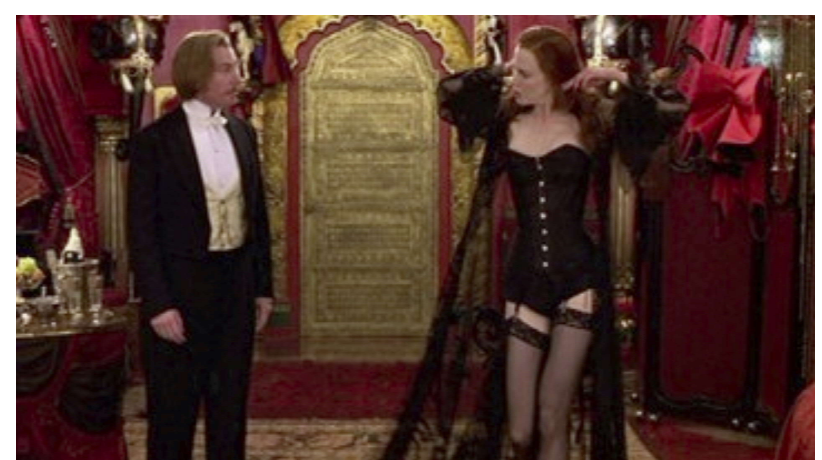

Foto 2. El duque (Richard Roxburgh) excomulgado por Satine.

Satine es internacional. Satine es la bailarina Salome que seduce a su padrastro banquero, a su profesor de religión, a los padres de sus amigas del colegio o a los clientes donde hace strip-tease. Es la madre de soltera de Helen o los huérfanos abusados sexualmente ${ }^{10}$. Es Fantine defendiendo la supervivencia de Cosette ante la férrea persecución de Javert ${ }^{11}$.

Satine es la cubana o cubano a la caza de pepes y pepas para intentar vivir mejor de lo material a pesar de su comprometimiento moral muchas veces ajeno a la habitual crianza de la familia cubana.

No podemos soslayar el tratamiento a enfermedades que se abordan como la narcolepsia y la tuberculosis los que posibilitan explicar su etiología, diagnóstico y tratamiento partiendo de la génesis de su tratamiento en la época comparados con la actualidad e introducir los resultados alentadores de la medicina en el país $12,13,14$.

A esto se suma la banda sonora de la película que expone temas musicales pop y rock de los años 80 y 90 que pueden convertirse en germen de iluminación para los jóvenes futuros galenos para los cuales muchos son temas desconocidos. Clásicos de Elton John como Your Song; The Show Must Go On de Queen; Like a Virgin de Madonna y la presencia de otros exponentes como David Bowie, Sting, engendran un emporio digno de admirar y deleitar máxime cuando en los gustos en la actualidad se enmarcan en el reguetón fácil y vulgar, aunque haya músicos que apuestan por una letra mejor perfilada.

Un aparte merece el tratamiento musical al tango, género poco apreciado entre los jóvenes, rejuvenecido con una frescura y violencia metódica expuesto con fineza y fuerza extraordinaria lo que favorece la labor con los discípulos aficionados a la manifestación de danza y que ha suscitado no pocas ideas novedosas.
Las propuestas de proyectos alternativos de terapia musical pueden motivarse con esta propuesta tanto para visualizar o bailarla además de servir para resaltar lo tradicional no solamente de Cuba sino de otras regiones representadas o no en el colectivo estudiantil extranjero en el centro y también a la pedagogía médica cubana como integrante inexorable de la cultura.

En fin, es preciso que los educadores ensayen con materiales fílmicos videos o documentales con el objetivo de apoyar con recursos novedosos en la preparación de los futuros médicos. Esta cinta en particular provee de recursos al docente para contribuir a la formación cultural complementaria de los estudiantes de Medicina a través del cine motivando su gusto por el buen arte y las posibilidades de vinculación con la profesión.

\section{Referencias}

1. García Torres DS, Sánchez Hechavarría M, Montoya Ledel M, Franco Díaz C, Díaz Suárez R. El Cine Club como vía para el debate y tratamiento a la educación preventiva antidrogas en estudiantes de medicina. Rev Med Cine 2016; 12(4):236-9.

2. Moreta Velayos Fl; Moreta Montero C; Montero Sánchez N; de la Fuente Hermosín I. Sesión de cine en atención primaria. Rev Med Cine 2016; 12(1): 6063.

3. Moratal Ibáñez LM. Medicina y Cine o el Cine como Medicina. Rev Med Cine 2006; 2(3): 77-9.

4. Jiménez Carabias MR, Vidal Salcedo A, Carmena Hernández A I. El cine, una herramienta útil para la docencia. II Conferencia Internacional de Comunicación en Salud. Universidad Carlos III de Madrid. 23 de octubre de 2015.

5. García Torres DS, Fuentes Domínguez N, Mendoza Ruiz M, Díaz Suárez R, Perera Díaz J. El cine debate con estudiantes de medicina para incidir preventivamente sobre el sexo transaccional. Rev Med Cine 2018;14 (en prensa).

6. Garrido Amable O, Garrido Amable G, Amable Ambrós ZM. Cultura de salud en estudiantes de Medicina desde la perspectiva curricular. Educ Med Super. 2015;29(1): 72-7.

7. Berovides Álvarez V, Alfonso Sánchez MA. Genoma, cultura y evolución. La Habana: Editorial Ciencia y Técnica; 2007.

8. Hernández Figaredo P, Peña García F. El cine como herramienta en la docencia de Psiquiatría. Rev Humanidades Médicas 13(1):1-16.

9. Cañete Rojas Y. Necesidad social de la cultura socio-médica del especialista en medicina general integral desde la superación profesional. MULTIMED Granma 2015; 19 (supl 1).

10. Atwood M. El quetzal resplandeciente y otros relatos. La Habana : Editorial Arte y Literatura; 2009.

11. Victor Hugo. Los miserables.

12. Pabón RM, García de Gurtubay I, Morales G, Urriza J, Imirizaldu L, Ramos Argüelles F. Narcolepsia: actualización en etiología, manifestaciones clínicas y tratamiento. Anales Sis San Navarra 201033 (2): 191-201.

13. González Ochoa E, González Díaz A, Armas Pérez L, Llanes Cordero MJ, Marrero Figueroa A, Suárez Alvarez $L$ et al. Tendencia de la incidencia de Tuberculosis en Cuba: lecciones aprendidas en 1991-1994 y su transcendencia en 2004-2012. Rev Cubana Med Trop 2015; 67(1): 122-138.

14. Grave de Peralta YT, Lafargue Mayoz D, Montero Migota M, Curí Quevedo S, Campos Muñoz M. Tuberculosis: tendencia, pronóstico y factores de riesgo afines en la provincia de Santiago de Cuba (2004-2014). MEDISAN 2016 ;20(4):455461. 